\title{
Interference Aware (IA) MAC: an Enhancement to IEEE802.11b DCF
}

\author{
Matteo Cesana ${ }^{\S *}$, Daniela Maniezzo*†, Pierpaolo Bergamo ${ }^{\dagger}$, Mario Gerla* \\ ${ }^{\S}$ Dipartimento di Elettronica e Informazione, Politecnico di Milano, Italy \\ ${ }^{*}$ Computer Science Department - ${ }^{\dagger}$ Electrical Engineering Department \\ University of California Los Angeles, Los Angeles, California, USA \\ cesana@elet.polimi.it,maniezzo@ee.ucla.edu,pbergamo@ee.ucla.edu,gerla@cs.ucla.edu
}

\begin{abstract}
The IEEE802.11 has been devised explicitly for low mobility and single access point scenarios, so its effectiveness is impaired in conditions with not negligible interference in ad hoc and/or in infrastructure modes. Well known problems typical of these scenarios are the so called hidden and exposed terminals. In this paper we propose and test a novel MAC layer for Wireless LANs, able to improve the performance of IEEE802.11 DCF (Distributed Coordination Function) in environments with high interference levels. The key point is to insert information about received power and interference levels into MAC control packets. By computing an estimation of the interference increasing due to an eventual transmission (forbidden with IEEE802.11 because blocked by the virtual carrier sensing), the number of parallel transmissions can grow significantly.
\end{abstract}

\section{INTRODUCTION}

The recent success of wireless technologies has boosted the development of wireless networking. Both industry and academia have turned their attention to this area, attracted initially by intriguing research issues and finally by market revenues.

The wireless environment proposes some challenging problems with which the network design has to cope. Firstly, the radio channel is prone to errors and temporary failures which are not encountered in the wired world; secondly, the channel is shared and resources are often scarce. In this scenario, the employed access control schemes are key points for achieving effectiveness.

Among the numerous standards for wireless communications, the IEEE802.11b [1] [2] is the one with the highest utilization. IEEE802.11 places specifications on the parameters of both the physical (PHY) and medium access control (MAC) layers of the network. The physical layer covers the physical interface. IEEE802.11b physical layer is an extension to IEEE802.11 physical layer that provides three options: Infrared baseband, Frequency Hopping Spread Spectrum and Direct Sequence Spread Spectrum in the $2.4 \mathrm{GHz}$ which support 1 and 2Mbit/s. IEEE802.11b can support higher data rates of 5.5 and $11 \mathrm{Mbit} / \mathrm{s}$ by using Complementary Code Keying (CCK) with Quadrature Phase Shift Keying (QPSK) modulation and Direct-Sequence Spread-Spectrum technology.

IEEE802.11 was originally devised explicitly for a single
Access Point scenario where all the mobile nodes are within the transmitting range of one another. In this environment, the IEEE802.11 medium access control protocol, which tends to avoid the interference, is able to achieve high efficiency. Problems arise when IEEE802.11 is used both in a pure ad hoc mode, where the basic IEEE802.11 mode is not able to exploit any spatial reuse, and in infrastructure cellular-like scenario where the connectivity is provided by different APs (Access Points) with overlapping transmission ranges, i.e., with non negligible interference [3].

The efficiency of IEEE802.11 based networks can be dramatically impaired by the well known hidden and exposed terminal problems [4]. As a matter of fact, the four-way handshake with the Virtual Carrier Sensing solves only partially the hidden terminal problem and, to the best of our knowledge, the exposed terminal is still a pitfall and can deeply affect the performance of multi-hop ad hoc networks based on IEEE802.11 [5].

Different solutions have been proposed in the literature to counteract these shortcomings of the IEEE802.11 standard. In particular, a major effort has been done in the development of an efficient medium access control protocol able to exploit spatial reuse and allowing parallel feasible communications [6]. The work on this topic deals primarily with the modification of the numerous timers the IEEE802.11 MAC layer [7] [8] with the purpose to achieve an efficient sharing of the common resource among all the users. Velayutham and Wang [9] proposed a distributed scheduling algorithm for IEEE802.11 networks able to solve the exposed terminal problem and consequently increase the spatial reuse. Recently, some works have appeared aiming at improving the IEEE802.11 MAC layer by exploiting the capture at the physical layer, that is to say the capacity of correctly receiving a transmission even if in presence of interfering communications [10] [11]. Like in the cellular systems, the capture effect is exploited in order to increase the reuse and consequently the bandwidth efficiency of the wireless medium [12].

Our work follows the second approach. In this paper we propose a novel MAC layer for Wireless LANs, named Interference Aware MAC (IA-MAC), reported in [13] and 
[14] even if in a slightly different version, which extends the capabilities of the basic IEEE802.11 in environments with high interference, both in ad hoc and in infrastructure mode [15] [16].

In details, we propose to enhance the information carried by the CTS packets (and in a more complex approach, also by the RTS packets) so that each transmitting/receiving node can estimate the interference it generates on all the other ongoing transmissions, and decides to transmit/receive if such an interference does not corrupt the others.

The basic principles of our mechanism are similar to the ones introduced in the Power Controlled Multiple Access (PCMA) proposed by Monks et al. [17]. Both protocols try to exploit the capture effect to increase the spatial reuse and, like PCMA, IA-MAC is based on a cooperation principle, i.e., no station is allowed to transmit/receive if its transmission/reception can destroy ongoing transmissions. On the other hand, our scheme differs from the PCMA in at least two significant aspects:

- IA-MAC does not implement any power control (our attention is focused on the access part rather than on the power management, which can be tricky in a distributed environment like the ad hoc one);

- PCMA uses an additional radio channel in order to transmit a busy tone with information on the interference levels, while in IA-MAC the estimation of the interference is done on the same channel used for data traffic; because of the last reason, IA-MAC seems to be more cost effective and better suited for wireless environments in which the battery consumption is a constraint.

This work is structured as follows: Section II summarizes the basics of the proposed IA-MAC protocol, by underlining the differences with respect to IEEE802.11; in Section III some simulation result are shown and the protocol performance are analyzed; finally, Section IV concludes the paper, by proposing future works.

\section{IA-MAC BASICS}

\section{A. Brief IEEE802.11 DCF Overview}

As far as IEEE802.11 MAC layer is concerned, two different schemes are standardized: the Point Coordination Function (PCF) and the Distributed Coordination Function (DCF). While PCF is a centralized polling based access scheme, the DCF defines a distributed access algorithm based on the Carrier Sense Multiple Access with Collision Avoidance (CSMA/CA) protocol and it can be used in both infrastructure and ad hoc wireless LANs [1] [2].

DCF compliant nodes can use two access methods. The first one is based on a two-way handshake procedure (DATA/ACK), the second one adopts a four-way handshake procedure, where the DATA/ACK phase is preceded by a channel probing/acquiring phase called RTS/CTS (Request To Send/Clear

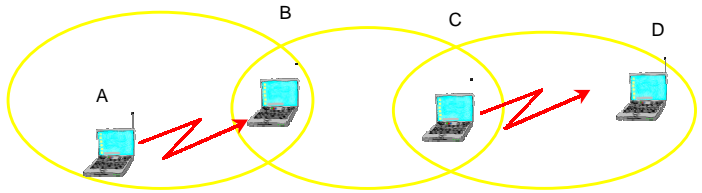

Fig. 1. A is sending a packet to B when $\mathrm{C}$ should decide whether to transmit to $\mathrm{D}$.

To Send). Basically, each node senses the channel before transmitting the first frame of the handshake. If the medium is idle for at least a DCF inter frame space (DIFS) period of time, the node starts transmitting the first frame of the handshake, otherwise the node waits for the channel to be idle for DIFS and draws a random additional backoff time to avoid possible collisions when the channel becomes free (Collision Avoidance). A further channel control mechanism is applied in the four-ways handshake procedure. According to this scheme, each control frame (RTS/CTS) brings information about the duration of the starting communication. Every other node overhearing that frame is prevented from accessing the channel for all the duration of the ongoing communication by setting a proper parameter called NAV (Network Allocation Vector). This procedure is usually called Virtual Carrier Sensing.

\section{B. IA-MAC Basic Version}

In IA-MAC, each node attempts to transmit always according to the DCF RTS/CTS access scheme. Furthermore, we require a very slight modification of the CTS packet which must convey further information used for the interference estimation phase. In details, each CTS packet carries:

- the experienced SINR (Signal to Interference and Noise Ratio) at the time of the reception of the RTS;

- the power level at which the RTS is received.

Since the quantities could be expressed in $d B m W(\mathrm{~dB}$ milliWatt), the values can be expressed in 1 byte for each field. These values are used by the node which overhears the CTS to decide whether to set or not the NAV, according to the rules described in the following. All the nodes overhearing a RTS packets (except for the recipient) set their NAV, as in the standard protocol. NAV is not always set on the reception of CTS. If a node receives a CTS, it decides whether to set its NAV or not on the basis of the information contained in the received CTS. In details, it runs an interference estimation procedure.

In order to understand better the protocol, let us consider four nodes (A, B, C, D) in a chain-like topology (see Figure 1). $\mathrm{A}$ is in radio coverage only with $\mathrm{B}, \mathrm{B}$ with $\mathrm{A}$ and $\mathrm{C}, \mathrm{C}$ with $\mathrm{B}$ and $\mathrm{D}$ and $\mathrm{D}$ only with $\mathrm{C}$. Let $\mathrm{A}$ have an ongoing communication toward B, i.e., A sent a RTS to B which answered with a CTS. Node C, upon reception of the CTS from B, estimates how much an own transmission could affect the reception of $\mathrm{B}$. 
Let $P_{B}^{R T S}$ be the power received by B on the RTS sent by A, and let $I_{B}^{r}$ be the sum of noise and interference perceived by B. B inserts in its CTS the information of $S I N R_{B}=P_{B}^{R T S} / I_{B}^{r}$ and $P_{B}^{R T S}$. C overhears the CTS sent by B. Let $P_{C}^{C T S}$ be the power $C$ receives on the CTS sent by $B$. In order to estimate the SINR B would get if $\mathrm{C}$ starts transmitting, $\mathrm{C}$ considers the channel symmetric, i.e., $\mathrm{B}$ would receive a packet from $\mathrm{C}$ with the same power $P_{C}^{C T S}$. Thus, $\mathrm{C}$ estimates that $\mathrm{B}$ would perceive a value of SINR equal to:

$$
S I N R_{B}^{\mathrm{ext}}=\frac{P_{B}^{R T S}}{I_{B}^{r}+P_{C}^{C T S}}=\frac{P_{B}^{R T S} S I N R_{B}}{P_{B}^{R T S}+P_{C}^{C T S} S I N R_{B}},
$$

since $I_{B}^{r}=P_{B}^{R T S} / S I N R_{B}$.

If $S I N R_{B}^{\text {ext }}$ is lower a certain threshold $(\gamma), \mathrm{C}$ refrains from transmitting by setting its NAV, otherwise it can send its own RTS.

The value of $\gamma$ depends on the sensitivity of the receiver and should be fixed in a conservative way in order to take into account the fact that the estimated interference could not be accurate for all the duration of the communication because of new eventual interferences.

\section{IA-MAC Improvements}

The results presented in this section refer to the Basic IAMAC implementation. No results are currently available about the improvements we are working on.

We are currently working on future improvements to the basic version of the protocol which will further increase the spatial reuse and consequently the overall gain with respect to the standard IEEE802.11. The basic idea is to relax the rule which wants the NAV to be set always upon reception of the RTS. We have found that in some situations the NAV could be not set even if the node has received a RTS. This can be done inserting the information on the interference level in the RTS as well, and exploiting the time diversity among parallel communications, that is to say cases where collision can be avoided because the nodes within the same coverage range are in the same transmitting/receiving state.

\section{Simulation Results}

In order to estimate the performance improvement that IAMAC reaches in ad hoc networks with respect of IEEE802.11, we developed a simulator. The simulator is IEEE802.11 DCF compliant, even though there is no power-saving feature. The simulator involves only physical and MAC layers. No routing protocols are considered, because only the performance at MAC layer are of interest in this work. We considered operative conditions already investigated in the literature. In particular, we considered the saturation condition, exploited in [18] [19], in which each source node has always a packet to be transmitted to a neighbor.

IA-MAC does not show any improvement if all the nodes are in radio range. In fact, in this case, each node receives any RTS sent by a transmitter and so each station sets its NAV. In order to outperform IEEE802.11, IA-MAC needs the presence of hidden terminals, i.e., some nodes are not in radio coverage each other. In this scenario, more parallel transmissions are possible and IA-MAC exploits this chance. In our results, we introduce the concept of "hidden terminal probability" $P_{h}$ used in [20] [21]. In our case, $P_{h}$ is the averaged percentage of terminals that are hidden from any other node, which is basically the ratio of the nodes in radio range of a terminal and the total number of nodes. The average is computed among all the nodes in the network. Another work that focuses on this topic is [22]. Since in this paper we show results only comparing IEEE802.11 and IA-MAC with performance ratios, the work [22] can be used to understand what the real performance of IEEE802.11 is.

\section{A. Simulator and Setup Parameters}

The simulator is able to perform IEEE802.11 communications. As required by IA-MAC, all the simulations consider to have RTS/CTS handshake at the beginning of the communication. The software we built simulates $N=50$ nodes uniformly distributed in a squared area with different side lengths. This is because we want to simulate different $P_{h}$ values and so different densities of the nodes. The nodes are stationary. Even though the interference level can vary if nodes are mobile, the time required for a transmission is not so long to impact the received power levels significantly. So, we consider this a good approximation. As discussed above, each source nodes is in saturation condition, which means that it has always a packet to be transmitted to a neighbor in radio coverage. The fraction of source terminals among the whole set of nodes is changed as a parameter of simulations. Each new packet has a different destination in the set of reachable neighbors. The packet payload size (at MAC layer) is constant and equal to $P_{s}=1 \mathrm{kB}$. We remark that, regardless the size of the packet, RTS/CTS handshake is always used also for IEEE802.11. The transmission bitrate on the channel is $B_{w}=1 \mathrm{Mbit} / \mathrm{s}$; the choice of $B_{w}$ is arbitrary but our scheme can be used with different bitrates, even though the value of $\gamma$ should change (dynamically, if adaptative rates are considered). Simulation time is $T=500$ s. Each result is the average obtained from several simulations.

As far as the threshold $\gamma$ is concerned, its value is obviously critic and maybe it is the parameter that mostly affects the performance. In all the simulations, we have fixed (in $\mathrm{dB}$ ):

$$
\gamma_{d B}=C E_{d B}+6_{d B}
$$

where $C E$ is the constant value of the Capture Effect, which means that a signal is correctly received if the ratio of the received power level and the sum of the interference and noise levels is greater than $C E$. Although the choice of $\gamma$ as in the formula above assures good performance in several conditions, 


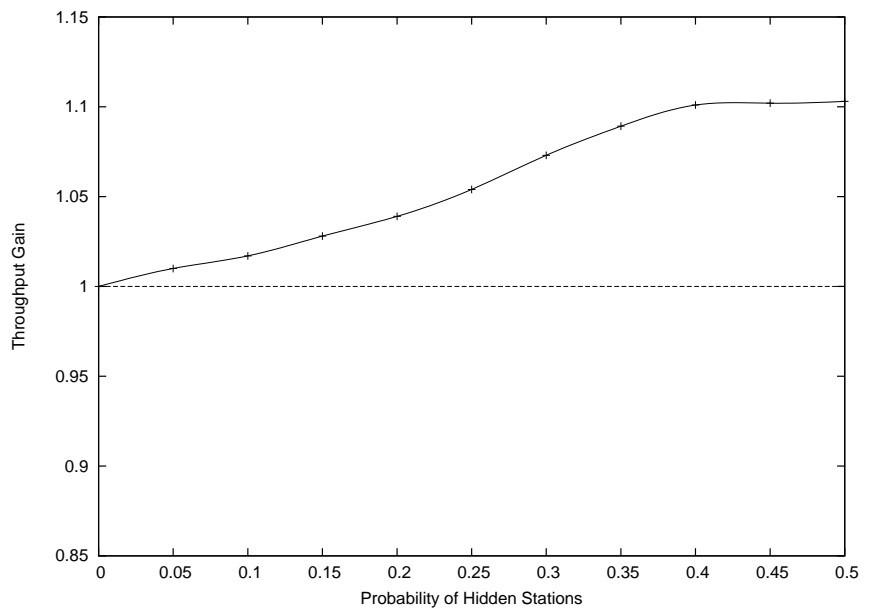

Fig. 2. Throughput as a function of hidden node probability $P_{h}(\mathrm{~S}=15$ source nodes).

this is an empiric value. Further analysis on this topic will be done in future works.

\section{B. Performance Indices}

In this work, we report two performance indices:

- throughput: the fraction of time in which the channel is occupied by a successful transmission of the MAC layer payload. In other words, the throughput $\mathrm{TH}$ is:

$$
T H=\frac{D_{b}}{B_{w} T}
$$

where $D_{b}$ is the total number of correctly delivered bits of MAC layer payloads;

- delay: the time required to deliver a packet, from its generation to the time in which the recipient receives the last bit of the packet. Because of the saturation condition in the packet generation process, a brief explanation about the generation time should be given. In this work, the generation of the new packet is timestamped at the same time that the previous packet acquires (or tries to acquire) the channel for the first time.

Since we are interested only in the performance improvement of IA-MAC with respect to IEEE802.11, the results show the throughput as the ratio of the throughput obtained by IA-MAC and the one obtained by IEEE802.11. It is a normalization that allows to understand very quickly the performance gain. The same ratio is to be considered for the delay. We will refer to these quantities as throughput gain and delay gain.

\section{Performance results}

In Figure 2, the throughput gain as a function of the hidden terminal probability $P_{h}$ is reported ( $S=15$ source nodes). As briefly outlined, when all the nodes are in radio coverage, IAMAC works and performs as IEEE802.11. In this scenario, the

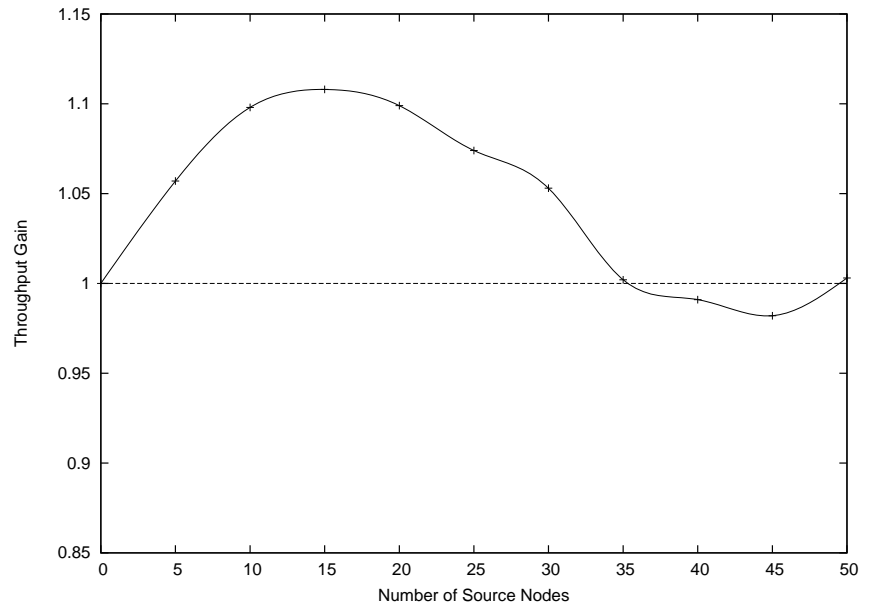

Fig. 3. Throughput as a function of source nodes $S\left(P_{h}=0.4\right)$.

throughput is almost the same, so the resulting throughput gain is equal to 1 . As far as $P_{h}$ is increased, our proposal shows an effective performance gain. The growth is almost linear with $P_{h}$. This is due to the increasing number of nodes that receive only the CTS and so, in some conditions, they are allowed to transmit to other neighbors. The growth is linear up to the maximum (about $10 \%$ at $P_{h}=0.4$ ). Beyond $P_{h}=0.4$ the performance increasing saturates. This is due to the fact that a lot of parallel transmissions occur and so the value of $\gamma$ is not sufficient to preserve the communication from interference. In Figure 2, only the values up to $P_{h}=0.5$ are reported. For higher values of $P_{h}$, the throughput of IEEE802.11 and of IA-MAC collapse (as reported in [22]) because of the number of collisions. Thus, a performance ratio in this condition has no meaning.

In Figure 3, the throughput gain as a function of number of source nodes $\left(P_{h}=0.4\right)$ is shown. When the traffic load is very low, ( $S$ close to 0 ), no improvement is expected by IA-MAC. Otherwise, when the load conditions increase, the NAV-setting is avoided often. Unfortunately this trend holds up to a certain value of $S(T H=11 \%$ at $S=$ 20 nodes). After that, the interference level is so high that sometimes the parallel transmissions that IA-MAC allows are corrupted. Nevertheless, it is just a reduction of the increasing of performance and not a reduction of performance. IA-MAC shows a performance loss only at very high values of $S$ but it should be noticed that it is negligible. We can underline that IA-MAC performs better than IEEE802.11 in almost all the operative conditions.

To confirm the effectiveness of IA-MAC, results concerning with delivery delay gain are shown in Figure 4. When IA-MAC mechanism is working very well, i.e., for the lower values of $S$, the delivery time is smaller, which means that the system delivers the packets is more promptly. The trend is very similar to the throughput gain in Figure 3 (obviously, in this case there 


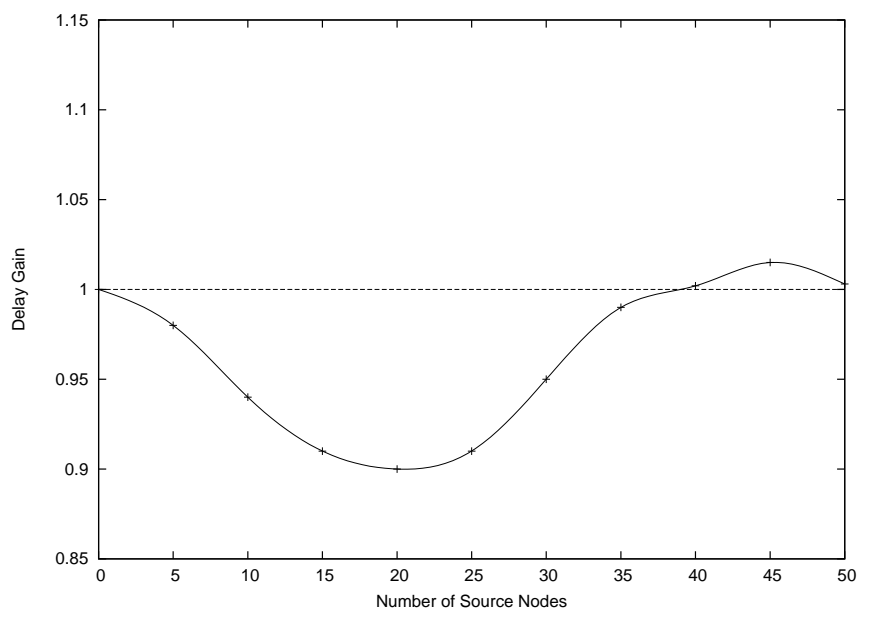

Fig. 4. Delay as a function of source nodes $S\left(P_{h}=0.4\right)$.

is a reduction of delay so the gain is lower than 1). When the CTS mechanism of IA-MAC starts to fail, also the delivery delay is affected because of the retransmissions. Also in this case, the performance of IA-MAC is better in almost all the simulation conditions and this demonstrates definitively the effectiveness of the idea.

\section{CONClusion AND Research in Progress}

In this paper we have presented an effective improvement to IEEE802.11 DCF MAC protocol. The proposed scheme, called Interference Aware MAC (IA-MAC), achieves a higher network spatial reuse by allowing feasible partially overlapping transmissions. To reach this goal we propose to include information about the Signal to Interference and Noise Ratio (SINR) and the received power levels into CTS header and to slightly modify the actual IEEE802.11 DCF.

We tested the effectiveness of the the proposed protocol by means of simulations. The results we obtained show that IAMAC grants a good gain in terms of throughput with respect to IEEE802.11 with low-medium network loads and grants lower packet delivery delays.

Further works will be addressed to evaluate the performance by varying the parameter $\gamma$. This value depends on the receiver sensitivity and can impact on the network spatial reuse and consequently on the overall performance.

Furthermore, we are working on two future improvements to IA-MAC; the former, by choosing whether to set NAV also after the reception of a RTS packet, taking into account the transmission durations; the latter just avoiding some collisions on the ACK packets (also in this case, exploiting the transmission durations).

\section{ACKNOWLEDGEMENTS}

This work has been partially funded by the UC Core program Core01-10091 under the sponsorship of ST Microelectronics. Corresponding Author: D. Maniezzo UCLA
Department of Computer Science, BH 3731, 420 Westwood

Plaza, Los Angeles, CA 90024, USA, dmaniezzo@ieee.org.

\section{REFERENCES}

[1] http://grouper.ieee.org/groups/802/11/

[2] IEEE Standard 802.11, Wireless LAN media access control (MAC) and physical layer (PHY) specifications, 1999.

[3] Haitao Wu, Shiduan Cheng, Yong Peng, Keping Long, Jian Ma, Does the IEEE 802.11 MAC protocol work well in multihop wireless ad hoc networks?, IEEE Communication Magazine, Volume 39, Issue 6, June 2001.

[4] Haitao Wu, Shiduan Cheng, Yong Peng, Keping Long, Jian Ma, IEEE802.11 distributed coordination function $(D C F)$ : analysis and enhancement, ICC 2002, New York, USA, April 2002.

[5] C.C. Chow, V.C.M. Leung, Performance of IEEE 802.11 medium access control protocol over a wireless local area network with distributed radio bridges, WCNC 1999, New Orleans, LA, USA, September 1999.

[6] J. Deng and Z. Haas, Dual busy tone multiple access (DBTMA)- a multiple access control scheme for ad hoc networks, IEEE Transactions on Communications, Volume 50, Issue 6, June 2002.

[7] V. Bharghavan, MACAW: a Media Access Protocol for Wireless LAN's, SIGCOMM 1994, London, UK, September 1994.

[8] F. Talucci, M. Gerla, L. Fratta, MACA-BI (MACA By Invitation)-a receiver oriented access protocol for wireless multihop networks, PIMRC 1997, Oulu, Finland, September 1997.

[9] A. Velayutham, H. Wang, Solution to the Exposed Node Problem of 802.11 in Wireless Ad-Hoc Networks, http://www.cs.iastate.edu/ vel/research/E-MAC.pdf.

[10] S.-L. Wu, Y.-C. Tseng, C.-Y. Lin and J.-P. Sheu, A Multi-Channel MAC Protocol with Power Control for Multi-Hop Mobile Ad Hoc Networks, Computer Journal, Volume 45, Issue 1, Jenuary 2002.

[11] J.So, N. Vaidya, A Multi-channel MAC Protocol for Ad Hoc Wireless Networks, Dept. of Electrical and Computer Engeneering, University of Illinois, USA, Technical Report, January 2003.

[12] T. Rappaport, Wireless Communications: Principles and Practice, Prentice Hall, New Jersey, USA, 1996.

[13] D. Maniezzo, M. Cesana, M. Gerla, IA-MAC: Interference Aware MAC for WLANs, UCLA Computer Science Department Technical Report 020037.

[14] D. Maniezzo, P. Bergamo, M. Cesana, M. Gerla, How to Outperform IEEE 802.11: Interference Aware (IA) MAC, MedHocNet 2003, Mahdia, Tunisia, June 2003.

[15] K.K. Leung, B. McNair, L.J. Cimini, J.H. Winters, Outdoor IEEE 802.11 cellular networks: MAC protocol design and performance, ICC 2002, New York, NY, USA, April 2002.

[16] P. Gupta, P. R. Kumar, The capacity of wireless networks, IEEE Transactions on Information Theory, Volume 46 Issue 2, March 2000.

[17] J.P. Monks, V. Bharghavan, W.-M.W. Hwu, A power controlled multiple access protocol for wireless packet networks, INFOCOM 2001, Anchorage, Alaska, April 2001.

[18] G. Bianchi, IEEE802.11 - Saturation Throughput Analysis, IEEE Communcation Letters, Volume 2, Issue 12, December 1998.

[19] G. Bianchi, Performance Analysis of the IEEE 802.11 Distributed Coordination Function IEEE Journal on Selected Areas in Communications, Volume 18, Issue 3, March 2000.

[20] K. C. Huang, K. C. Chen, Interference Analysis of Nonpersistent CSMA with Hidden Terminals in Multicell Wireless Data Networks, IEEE PIMRC 1995, Toronto, Canada, September 1995.

[21] T.S. Ho, K.C. Chen, Performance Analysis of IEEE 802.11 CSMA/CA Medium Access Control Protocol, IEEE PIMRC 1996, Taipei, Taiwan, ROC, October 1996.

[22] S. Rahman, Throughput Analysis of IEEE 802.11 Distributed Coordination Function in Presence of Hidden Stations, http://www.stanford.edu/class/ee384y/projects/download03/ shahriar2.pdf 